\title{
Black Women Are More Likely Than White Women to Schedule a Uterine-Sparing Treatment for Leiomyomas
}

\author{
Ganesa Wegienka, PhD, ${ }^{1}$ Elizabeth A. Stewart, MD, ${ }^{2}$ Wanda K. Nicholson, MD, MPH, ${ }^{3}$ Shuaiqi Zhang, MS, ${ }^{4}$ \\ Fan Li, PhD, ${ }^{5, *}$ Laine Thomas, PhD, ${ }^{4,5}$ James B. Spies, MD, ${ }^{6}$ Sateria Venable, BArch, ${ }^{7}$ \\ Shannon Laughlin-Tommaso, MD, MPH, ${ }^{2,8}$ Michael P. Diamond, MD, ${ }^{9}$ \\ Raymond M. Anchan, MD, ${ }^{10}$ George Larry Maxwell, MD, ${ }^{11}$ Erica E. Marsh, MD, MSCi, ${ }^{12}$ \\ Evan R. Myers, MD, MPH, ${ }^{13}$ Anissa I. Vines, PhD, ${ }^{14}$ Lauren A. Wise, ScD, ${ }^{15}$ \\ Kedra Wallace, $\mathrm{PhD}^{16}$ and Vanessa L. Jacoby, MD, MAS ${ }^{17}$
}

\begin{abstract}
Background: To evaluate differences in the proportion of uterine fibroid (UF) treatments that are uterinesparing between Black women and White women and identify factors that could explain disparities.

Methods: Women at age 18-54 years who were enrolled from 10 clinical sites in the United States into the Comparing Options for Management: Patient-Centered Results for UFs (COMPARE-UF) treatment registry completed questionnaires before their UF procedure. UF symptoms and quality of life were assessed by questionnaires. Details on UF imaging and treatment (hysterectomy, myomectomy, or uterine artery embolization [UAE]) were collected from each patient's medical record. Random-effects logistic regression was used to assess the association between race and the odds of having a uterine-sparing procedure versus hysterectomy. Subgroup analyses compared each uterine-sparing procedure with hysterectomy.

Results: In this cohort of 1141 White women and 1196 Black women, Black women tended to be younger (median 41.0 vs. 42.0 years) and report worse symptoms, pain, and function on every scale compared with White women. Black women were more likely to have had a prior UF treatment compared with White women $(22.8 \%$ vs. $14.6 \%)$. White women had more hysterectomies (43.6\% vs. $32.2 \%)$ and myomectomies $(50.9 \%$ vs. $50.2 \%)$ versus Black women. Black women had more UAEs (15.1\% vs. 4.7\%) than White women. After adjusting for clinical site and other variables, Black women had greater odds than White women of having a myomectomy (odds ratio $[\mathrm{OR}]=2.41,95 \%$ confidence interval $[\mathrm{CI}]=1.63-3.56$ ) or a UAE versus hysterectomy $(\mathrm{OR}=4.24,95 \% \mathrm{CI}=2.41-7.46)$.

Conclusion: In these participants, Black women were more likely to schedule a uterine-sparing UF treatment and a nonsurgical UF treatment than their White counterparts; this may not be true for all women. Longer

\footnotetext{
${ }^{1}$ Department of Public Health Sciences, Henry Ford Health System, Detroit, Michigan, USA.

${ }^{2}$ Department of Obstetrics and Gynecology, Mayo Clinic, Rochester, Minnesota, USA.

${ }^{3}$ Department of Obstetrics and Gynecology, Center for Women's Health Research, and Center for Health Promotion and Disease

${ }^{4}$ Duke Clinical Research Institute, Duke University School of Medicine, Durham, North Carolina, USA.

${ }^{5}$ Department of Biostatistics and Bioinformatics, Duke University School of Medicine, Durham, North Carolina, USA.

${ }^{6}$ Department of Radiology, Georgetown University School of Medicine, Washington, District of Columbia, USA.

${ }^{7}$ The Fibroid Foundation, Bethesda, Maryland, USA.

${ }^{8}$ Department of Surgery, Mayo Clinic, Rochester, Minnesota, USA.

${ }^{9}$ Department of Obstetrics and Gynecology, Augusta University, Augusta, Georgia, USA.

${ }^{10}$ Department of Obstetrics, Gynecology and Reproductive Biology, Brigham and Women's Hospital, Boston, Massachusetts, USA.

${ }^{11}$ Department of Obstetrics and Gynecology, Inova Fairfax Hospital, Falls Church, Virginia, USA.

${ }_{12}^{12}$ Department of Obstetrics and Gynecology, University of Michigan, Ann Arbor, Michigan, USA.

${ }^{13}$ Department of Obstetrics and Gynecology, Duke University School of Medicine, Durham, North Carolina, USA.

${ }^{14}$ Department of Epidemiology, Gillings School of Global Public Health, University of North Carolina, Chapel Hill, North Carolina, USA.

${ }^{15}$ Slone Epidemiology Center, Boston University School of Public Health, Boston, Massachusetts, USA.

${ }^{16}$ Department of Obstetrics and Gynecology, University of Mississippi Medical Center, Jackson, Mississippi, USA.

${ }^{17}$ Department of Obstetrics, Gynecology and Reproductive Sciences, University of California, San Francisco, California, USA.

*Current affiliation: Department of Biostatistics, Yale School of Public Health, New Haven, Connecticut, USA.
} Prevention, University of North Carolina, Chapel Hill, North Carolina, USA.
\end{abstract}


comparative effectiveness studies are needed to inform women about the durability of UF treatments. Greater understanding of factors influencing treatment selection is needed as are studies that include women without access to tertiary care centers. Clinical Trial Registration: Clinicaltrials.gov, NCT02260752 (enrollment start: November 2015).

Keywords: uterine fibroids, racial differences, hysterectomy, myomectomy, uterine artery embolization

\section{Introduction}

B Y MENOPAUSE, MOST women will have developed uterine leiomyomas (fibroids). ${ }^{1}$ Symptomatic uterine fibroids (UFs) can cause heavy menstrual bleeding, pelvic pain and pressure, dyspareunia, and a bulky abdominal appearance. ${ }^{2}$ UF can also adversely affect fertility and birth outcomes, although these data are limited. ${ }^{3}$ Despite the large public health burden of UF in the United States, little is known about UF natural history or pathogenesis owing to a lack of longitudinal studies with imaging to confirm UF status. However, it is well accepted that the strongest risk factor for having UF is being of Black race. ${ }^{4}$ Other suspected risk factors include increasing age up to time of menopause and greater time since last giving birth. ${ }^{4}$ Three-quarters of the UF treatments in the United States are hysterectomies; however, uterine-sparing procedures are becoming more common as their clinical availability has increased. ${ }^{4}$ Common uterinesparing procedures include myomectomy (fibroid removal), uterine artery embolization (UAE), and endometrial ablation (EA). Those who have these uterine-sparing treatments, however, may require additional UF treatment over time for persistent symptoms or recurrent or new UF.

Black women are more likely than White women to develop UF, with more than $80 \%$ of Black women and nearly $70 \%$ of White women having ultrasound-based evidence of UF by menopause. ${ }^{1}$ Black women also tend to have a greater number and larger size UF at the time of diagnosis, typically diagnosed at younger ages relative to White women. ${ }^{5}$ The etiology of these racial disparities is unknown. Reasons for this may include greater provider vigilance for identification and treatment in Black women because of the higher prevalence. As a result, Black women may find themselves seeking a UF treatment at younger ages than their White counterparts. Stewart et al. have previously reported that among women with symptomatic UF, Black women are more likely than White women to report a preference for uterine-sparing fibroid treatments. $^{6}$

Using the national UF treatment registry, Comparing Options for Management: Patient-Centered Results for UFs (COMPARE-UF), we evaluated the extent to which the proportion of UF treatments that were uterine-sparing differed between Black women and White women. ${ }^{7,8}$ We also investigated potential reasons for differences between Black and White women's choices of procedural intervention.

\section{Methods}

The COMPARE-UF registry is under the direction of the Duke Clinical Research Institute, which served as the datacoordinating center for enrollment and follow-up. Clinical sites for this multisite registry across the United States are:
Brigham and Women's Hospital, Henry Ford Health System, INOVA Health System, the Mayo Clinic Network, the University of California Fibroid Network, the University of Michigan, the University of Mississippi Medical Center, and the University of North Carolina at Chapel Hill. Additional participants who planned to have a UAE were recruited from two specialty clinics at the Atlanta Fibroid Center and Georgetown University. IRB approval was obtained. All participants provided informed consent. ${ }^{7}$

In brief, women scheduled for an UF treatment at any of these sites were invited to complete a baseline survey on their health and quality of life, including the disease-specific validated Uterine Fibroid Symptom and Quality of Life (UFS-QOL) instrument, ${ }^{9-11}$ the EuroQOL 5-dimension with visual analog scale for overall wellness, ${ }^{12}$ a financial toxicity questionnaire, ${ }^{13}$ and the Patient Health Questionnaire- $2^{14}$ to screen for depression. Women self-reported their race. Women also answered questions about their reproductive health history, demographic characteristics including their insurance type, and any prior UF treatments. Medical chart review, performed by a centralized team of credentialed Registered Health Information Technicians at Henry Ford Health System, was conducted for each participant's baseline treatment. UF characteristics (estimated total UF volume, estimated uterine volume, and number of UF) based on pretreatment imaging were taken from the medical chart as were details of the treatment that was performed. Women who were 18-54 years of age at the time of UF treatment were recruited into the study. Although the study participants were of varying races and ethnicities, there were sufficient numbers of Black and White women to assess racial differences between these groups. Women were not excluded based on reported desire for future fertility. Black and White women who had either an UAE, a hysterectomy, or a myomectomy by any approach were included in these analyses. UAE and myomectomy were classified as uterinesparing procedures.

Participants whose planned treatment was an EA or MRIguided focused ultrasound (MRgFUS) were excluded from the main analyses as these procedures were only available at a minority of the recruitment sites. There were 193 EAs performed (56\% for Black women) and $166(86.0 \%)$ were performed at Henry Ford Health System, 8 were performed at the University of Mississippi, 3 sites performed none and no other site performed more than 5. There were $19 \mathrm{MRgFUS}$ performed-18 at the Mayo Clinic and one at Henry Ford Health System and 79\% were performed for White women. We have included a Appendix Table A1 in which these two procedures were added to the final models for purposes of sensitivity analyses. Women who were using medical management for their UF were not included in these analyses. Choosing between either initiating/continuing medical 
management or having a procedural intervention like the women in these analyses is a different decision-making process compared with selecting a specific procedure.

Variables considered as potential confounders were those we have previously used in our analyses. ${ }^{15,16}$ These variables include age, insurance type (public, private, or military), financial toxicity (rather than income and education), time since being diagnosed with UF, body mass index (BMI) (continuous $\mathrm{kg} / \mathrm{m}^{2}$ ), current use of birth control (any birth control pill, patch, ring, implant, intrauterine device, or injectable), whether the participant had any prior procedures for UF treatment, discomfort during intercourse, whether the participant's menstrual periods were "regular" or "predictable," frequent urination, previous number of pregnancies, current marijuana use, calculated total fibroid volume, calculated uterine volume, and number of fibroids identified. Indicator variables were also created for self-report of each of the following health conditions (comorbidities): high blood pressure, diabetes, asthma, thyroid problems, and blood clots in the legs or lungs. Self-report (yes/no) of the following were also included in the analyses: having menstrual periods that lasted 7 or more days, heavy bleeding during periods, and bleeding between periods.

Counts and percentages, as well as medians with interquartile ranges, were used to describe the data. To compare factors between race groups, we used Pearson chi-square tests for categorical variables and chi-square rank-based group means score test statistics for continuous variables. We then used random-effects logistic regression to sequentially adjust for patient-level demographic and health variables, and socioeconomic status (SES) represented by the financial toxicity score, as well as clinical site. We then tested the significance of the adjusted odds ratio (OR) in the randomeffects model. The significance test for the variance component of the site random effects helps assess whether clinical site is an important driving factor for differences in procedure rates. ORs and $95 \%$ confidence intervals (CIs) were generated to assess the association between race and the odds of having a uterine-sparing procedure versus a hysterectomy. Subgroup analyses were then performed to specifically consider UAE versus hysterectomy, and myomectomy versus hysterectomy.

\section{Results}

The cohort for this analysis comprised 1141 White women and 1196 Black women. Baseline patient characteristics are presented by race group in Appendix Table A2. In brief, the groups significantly differed statistically for every characteristic examined, including age, BMI, symptoms, and UFSQOL, except the visual analog score for wellness, which was 75.0 for both groups. Black women tended to be younger (median age 41.0 vs. 42.0 years overall) and to report worse symptoms, pain, and function on every scale compared with White women. Black women were also more likely to have had a previous procedure for UF treatment compared with White women $(22.8 \%$ vs. $14.6 \%)$.

Tables 1 and 2 provide the proportions of uterine-sparing procedures planned by site and by treatment separately for White women and Black women. Except at the University of Mississippi Medical Center, White women had lower percentages of uterine-sparing treatments compared with Black
Table 1. Proportions of Uterine-Sparing Procedures Planned by Site and by Treatment

\begin{tabular}{|c|c|c|}
\hline Site & White & Black \\
\hline $\begin{array}{l}\text { Brigham and Women's } \\
\text { Hospital }\end{array}$ & $72 / 92(78.3)$ & $47 / 51(92.2)$ \\
\hline INOVA Health System & $27 / 90(30.0)$ & $53 / 95(55.8)$ \\
\hline Henry Ford Health System & $47 / 88(53.4)$ & $193 / 246(78.5)$ \\
\hline Mayo Clinic Network & $100 / 257(38.9)$ & $21 / 26(80.8)$ \\
\hline University of Michigan & $37 / 70(52.9)$ & $25 / 42(59.5)$ \\
\hline $\begin{array}{l}\text { University of California } \\
\text { Fibroid Network }\end{array}$ & $201 / 246(81.7)$ & $74 / 86(86.1)$ \\
\hline $\begin{array}{l}\text { University of Mississippi } \\
\text { Medical Center }\end{array}$ & $18 / 33(54.6)$ & $86 / 203(42.4)$ \\
\hline $\begin{array}{l}\text { University of North } \\
\text { Carolina at Chapel Hill }\end{array}$ & $73 / 149(48.9)$ & $211 / 319(66.1)$ \\
\hline Atlanta Fibroid Center ${ }^{\mathrm{a}}$ & 4/4 (100) & $31 / 31(100)$ \\
\hline Georgetown University $^{\mathrm{a}}$ & 4/4 (100) & $12 / 12(100)$ \\
\hline
\end{tabular}

Data shown are number of women with uterine-sparing UF treatment/all women who had a UF treatment (\%).

${ }^{a}$ Only UAE patients were recruited from these sites.

UAE, uterine artery embolization; UF, uterine fibroid.

women. Overall, White women had more hysterectomies (43.6\% vs. $32.2 \%)$ and myomectomies (50.9\% vs. $50.2 \%)$ than Black women, and Black women had more UAEs (17.6\% vs. $5.5 \%$ ) (Table 2).

Baseline characteristics are compared between the White women and Black women by planned procedure in Table 3 (hysterectomy), Table 4 (myomectomy), and Table 5 (UAE). Regardless of the planned procedure, Black women tended to be younger and have larger BMIs. For women who planned to have a hysterectomy, Black women tended to have more UF and greater UF volume, but this was not true for women who planned to have an UAE. Black women who planned a myomectomy tended to have more UF but not greater UF volume. Among those who planned a hysterectomy or myomectomy, Black women were also more likely to have had a prior UF treatment; however, this was not true for women who planned UAE.

Black women were more likely to report having menses for at least 7 days compared with White women regardless of the planned treatment. Among women who planned a hysterectomy or myomectomy, Black women were also more likely to have reported pelvic pain requiring medication. Black women planning a myomectomy were more likely than White women planning a myomectomy to have reported heavy menses.

Table 2. Procedure by Race Group

\begin{tabular}{lcc}
\hline Procedure & $\begin{array}{c}\text { White } \\
(\mathrm{N}=1033)\end{array}$ & $\begin{array}{c}\text { Black } \\
(\mathrm{N}=1112)\end{array}$ \\
\hline $\begin{array}{l}\text { Hysterectomy } \\
\text { Uterine-sparing }\end{array}$ & $450(43.6)$ & $358(32.2)$ \\
$\quad$ procedure & $583(56.4)$ & $753(67.8)$ \\
$\quad$ Myomectomy & $526(50.9)$ & $557(50.2)$ \\
UAE & $57(5.5)$ & $196(17.6)$ \\
\hline
\end{tabular}

Data are given as $n(\%)$.

Chi-square test comparing the rate of the procedure versus hysterectomy between races, $p<0.05$. 
Table 3. Baseline Characteristics of Women With a Planned Hysterectomy

\begin{tabular}{|c|c|c|c|}
\hline Characteristic & White $(\mathrm{N}=450)$ & Black $(\mathrm{N}=358)$ & $\mathrm{p}^{\mathrm{a}}$ \\
\hline Age (years) & $46.0(42.0,49.0)$ & $44.0(41.0,47.0)$ & $<0.05$ \\
\hline Insurance & & & $<0.05$ \\
\hline Private & $404(89.8)$ & $253(70.7)$ & \\
\hline Active military & $2(0.4)$ & $2(0.6)$ & \\
\hline Other & $44(9.8)$ & $103(28.8)$ & \\
\hline BMI $\left(\mathrm{kg} / \mathrm{m}^{2}\right)$ & $27.7(24.2,33.0)$ & $33.4(28.7,39.1)$ & $<0.05$ \\
\hline Financial toxicity $($ lower score $=$ worse status $)$ & $30.0(23.0,36.0)$ & $24.0(15.0,31.0)$ & $<0.05$ \\
\hline Duration of symptoms (years) & $3.0(1.0,8.0)$ & $5.0(2.0,12.0)$ & $<0.05$ \\
\hline Any bleeding symptoms & $375(83.9)$ & $306(85.7)$ & 0.48 \\
\hline Menses $\geq 7$ days & $254(56.8)$ & $229(64.1)$ & 0.04 \\
\hline Heavy menses & $344(77.0)$ & $284(79.6)$ & 0.38 \\
\hline Bleeding between periods & $232(51.9)$ & $191(53.5)$ & 0.65 \\
\hline Frequent urination & $263(58.8)$ & $218(61.1)$ & 0.52 \\
\hline Discomfort during intercourse & $193(43.2)$ & $174(48.7)$ & 0.12 \\
\hline Pelvic pain requiring meds & $209(46.8)$ & $201(56.3)$ & $<0.05$ \\
\hline Pelvic pain not during periods & & & $<0.05$ \\
\hline Pelvic pain during periods & $66(14.8)$ & $48(13.4)$ & \\
\hline No pelvic pain & $241(53.9)$ & $160(44.8)$ & \\
\hline Current contraception & $313(69.6)$ & $224(62.6)$ & $<0.05$ \\
\hline $\begin{array}{l}\text { Previous medical condition (high blood pressure, } \\
\text { diabetes, asthma, thyroid problems, blood clots in legs) }\end{array}$ & $224(50.6)$ & $217(61.6)$ & $<0.05$ \\
\hline Marijuana & $27(6.1)$ & $27(7.7)$ & 0.11 \\
\hline Any prior UF treatment & $82(18.4)$ & $83(23.5)$ & 0.08 \\
\hline Regular, predictable menses & $263(59.2)$ & $168(48.1)$ & $<0.05$ \\
\hline Number of previous pregnancies & & & $<0.05$ \\
\hline 0 & $120(27.0)$ & $53(15.1)$ & \\
\hline 1 & $62(13.9)$ & $44(12.5)$ & \\
\hline 2 & $98(22.0)$ & $60(17.0)$ & \\
\hline 3 & $81(18.2)$ & $63(17.9)$ & \\
\hline 4 & $38(8.5)$ & $63(17.9)$ & \\
\hline 5 & $23(5.2)$ & $30(8.5)$ & \\
\hline 6 & $11(2.5)$ & $16(4.5)$ & \\
\hline$>6$ & $12(2.7)$ & $23(6.5)$ & \\
\hline Total UF volume $\left(\mathrm{cm}^{3}\right)$ & $123.2(28.7,281.9)$ & $139.7(43.1,328.9)$ & 0.05 \\
\hline Uterine volume $\left(\mathrm{cm}^{3}\right)$ & $325.6(177.1,601.0)$ & $550.7(272.6,1037.5)$ & $<0.05$ \\
\hline Number of UFs measured & $2.0(1.0,3.0)$ & $3.0(1.0,4.0)$ & $<0.05$ \\
\hline Concern ${ }^{\mathrm{b}}$ & $40.0(20.0,60.0)$ & $25.0(10.0,45.0)$ & $<0.05$ \\
\hline Activities $^{\mathrm{b}}$ & $46.4(28.6,71.4)$ & $35.7(17.9,60.7)$ & $<0.05$ \\
\hline Energy/mood ${ }^{b}$ & $46.4(28.6,64.3)$ & $35.7(16.2,57.3)$ & $<0.05$ \\
\hline Control $^{\mathrm{b}}$ & $50.0(34.4,69.4)$ & $40.0(20.0,65.0)$ & $<0.05$ \\
\hline Self-conscious ${ }^{\mathrm{b}}$ & $41.7(16.7,66.7)$ & $33.3(8.3,58.3)$ & $<0.05$ \\
\hline Sexual function ${ }^{\mathrm{b}}$ & $50.0(25.0,75.0)$ & $37.5(12.5,62.5)$ & $<0.05$ \\
\hline Total-sum of 6 subscale scores above ${ }^{b}$ & $44.8(30.0,62.9)$ & $36.2(18.1,54.3)$ & $<0.05$ \\
\hline $\begin{array}{l}\text { Symptom severity (lower score indicates } \\
\text { more positive health status) }\end{array}$ & $59.4(43.8,75.0)$ & $68.8(50.0,84.4)$ & $<0.05$ \\
\hline Mobility & & & $<0.05$ \\
\hline I have no problems in walking about & $360(80.4)$ & $232(65.7)$ & \\
\hline I have some problems in walking about & $56(12.5)$ & $64(18.1)$ & \\
\hline I have moderate problems in walking about & $26(5.8)$ & $42(11.9)$ & \\
\hline I have severe problems in walking about & $6(1.3)$ & $14(4.0)$ & \\
\hline I am confined to bed & $0(0.0)$ & $1(0.3)$ & \\
\hline Self-care & & & 0.06 \\
\hline I have no problems washing or dressing myself & $423(94.6)$ & $321(90.4)$ & \\
\hline I have slight problems washing or dressing myself & $17(3.8)$ & $19(5.4)$ & \\
\hline I have moderate problems washing or dressing myself & $6(1.3)$ & $11(3.1)$ & \\
\hline I have severe problems washing or dressing myself & $0(0.0)$ & $3(0.8)$ & \\
\hline I am unable to wash or dress myself & $1(0.2)$ & $1(0.3)$ & \\
\hline Usual activities & & & 0.15 \\
\hline I have no problems doing my usual activities & $282(62.8)$ & $204(57.6)$ & \\
\hline I have slight problems doing my usual activities & $94(20.9)$ & $76(21.5)$ & \\
\hline I have moderate problems doing my usual activities & $56(12.5)$ & $47(13.3)$ & \\
\hline I have severe problems doing my usual activities & $14(3.1)$ & $20(5.6)$ & \\
\hline I am unable to perform my usual activities & $3(0.7)$ & $7(2.0)$ & \\
\hline
\end{tabular}


TABle 3. (CONTINUED)

\begin{tabular}{lccc}
\hline Characteristic & White $(\mathrm{N}=450)$ & Black $(\mathrm{N}=358)$ & $\mathrm{p}^{\mathrm{a}}$ \\
\hline Pain/discomfort & & & $<0.05$ \\
I have no pain or discomfort & $79(17.6)$ & $48(13.5)$ & \\
I have slight pain or discomfort & $151(33.7)$ & $71(20.0)$ & \\
I have moderate pain or discomfort & $155(34.6)$ & $131(36.9)$ & \\
I have severe pain or discomfort & $51(11.4)$ & $74(20.8)$ & \\
I have extreme pain or discomfort & $12(2.7)$ & $31(8.7)$ & \\
Anxiety/depression & $158(35.4)$ & $146(41.0)$ & $<0.05$ \\
I am not anxious or depressed & $170(38.1)$ & $98(27.5)$ & \\
I am slightly anxious or depressed & $91(20.4)$ & $65(18.3)$ & \\
I am moderately anxious or depressed & $23(5.2)$ & $29(8.1)$ & \\
I am severely anxious or depressed & $4(0.9)$ & $18(5.1)$ & \\
I am extremely anxious or depressed & $74.0(54.0,85.0)$ & $75.0(57.0,84.0)$ & 0.33 \\
Visual Analogue Scale (0-100) & & &
\end{tabular}

Data are median (interquartile range) or $n(\%)$.

${ }^{\mathrm{a}} p$-Values are based on Pearson chi-square tests for all categorical row variables. $p$-Values are based on chi-square rank based group means score statistics for all continuous/ordinal row variables. All tests treat the column variable as nominal.

${ }^{b}$ Higher score indicates more positive health status.

BMI, body mass index.

In the adjusted analyses, the site-level variances were statistically significantly different from 0 in all models (Table 6), indicating that site was associated with the treatment received. After adjusting for UF characteristics, clinical site, demographics, health, and other variables, Black women had greater odds than White women of having a myomectomy versus hysterectomy $(\mathrm{OR}=2.41,95 \% \mathrm{CI}=1.63-3.56)$ or a $\mathrm{UAE}$ versus hysterectomy $(\mathrm{OR}=4.24,95 \% \mathrm{CI}=2.41-7.46)$ (Table 6$)$. The sensitivity analyses including EA and MRgFUS among the uterine-sparing procedures yielded similar results to the primary analysis. The CIs of both analyses largely overlap and the point estimates have similar direction and magnitude (Appendix Table A2).

\section{Discussion}

In this analysis from a large multisite U.S. registry of women who planned treatment for their UF, Black women were more likely than their White counterparts to have either a myomectomy or UAE compared with hysterectomy after adjustments for UF characteristics, symptoms, previous UF treatment, and financial toxicity associated with the treatment. Furthermore, these data show that Black women were more likely to schedule a nonsurgical treatment (UAE) compared with White women. These results align with the work of Stewart et al. showing that Black women with symptomatic UF reported a preference for uterine-sparing UF treatments. ${ }^{6}$ These findings point to the importance of two high-priority research questions in the field of UF.

First, the reasons why women, and especially Black women, may seek uterine sparing treatment options is unknown and likely multifactorial. It may reflect distrust as a result of historic racial inequities in forced sterilization and hysterectomy. ${ }^{6,17,18}$ Choice of nonsurgical alternatives is also likely influenced by more rapid recovery than with a hysterectomy ${ }^{16}$ and by the patient's inability to take extended time off from work or need to manage child or elder care responsi- bilities. A woman's decision to keep her uterus may reflect her desire to maintain the possibility of childbearing, regardless of her actual fertility. This may be particularly true for the Black women who tended to be younger than the White women having an UF procedure. Among patients who underwent hysterectomy with or without oophorectomy, Farquhar et al. found that even 3 years after surgery, women regretted the loss of their fertility. ${ }^{19}$ Similarly, Leppert et al. reported that women who desired more children before hysterectomy tended to report higher levels of depression, anger, anxiety, and pelvic pain compared with women without this desire at $12-24$ months after surgery. ${ }^{20}$ Finally, for some women, the uterus is essential for "feminine" identity. Based on focus groups and personal interviews with African American women, $70 \%$ of whom had a hysterectomy, Augustus reported a general belief that women were no longer "whole" women after hysterectomy. ${ }^{21}$ This belief was also echoed in two recent focus group studies of both Black and White women who had undergone hysterectomy in Michigan and Alabama; these studies found that because of this belief, some women undergoing hysterectomy were afraid to share information about their surgery with others. ${ }^{22,23}$

The second research question introduced by this preference for uterine-sparing treatments, and often multiple sequential uterine-sparing treatments, is that Black women are at increased risk of future retreatment for recurrent and new fibroid symptoms. Stimulated by ovarian hormones, the risk of developing UF continues to menopause and prior research has shown that Black women do not have diminished growth as they reach this threshold as do White women. ${ }^{24,25}$ Understanding the individual clinical characteristics such as age, race/ethnicity, and baseline UF characteristics, which are predictors of success are critical to provide precision medicine approaches to treatment of UF. Because Black women are disproportionally affected by UF, ${ }^{8}$ the lack of understanding of predictors of subsequent treatment becomes a further 
Table 4. Baseline Characteristics of Women with a Planned Myomectomy

\begin{tabular}{|c|c|c|c|}
\hline Characteristic & White $(\mathrm{N}=526)$ & Black $(\mathrm{N}=557)$ & $\mathrm{p}^{\mathrm{a}}$ \\
\hline Age (years) & $38.0(33.0,43.0)$ & $37.0(33.0,42.0)$ & 0.07 \\
\hline Insurance & & & $<0.05$ \\
\hline Private & $464(88.7)$ & $440(79.4)$ & \\
\hline Active military & $2(0.4)$ & $10(1.8)$ & \\
\hline Other & 57 (10.9) & $104(18.8)$ & \\
\hline BMI $\left(\mathrm{kg} / \mathrm{m}^{2}\right)$ & $25.1(22.2,30.5)$ & $29.9(25.7,36.0)$ & $<0.05$ \\
\hline Financial toxicity (lower score $=$ worse status $)$ & $29.0(22.0,35.0)$ & $26.0(18.4,33.0)$ & $<0.05$ \\
\hline Duration of symptoms (years) & $2.0(1.0,5.0)$ & $4.0(1.0,10.0)$ & $<0.05$ \\
\hline Any bleeding symptoms & $409(77.8)$ & $462(83.1)$ & $<0.05$ \\
\hline Menses $\geq 7$ days & $267(50.8)$ & $317(57.0)$ & $<0.05$ \\
\hline Heavy menses & 378 (71.9) & 435 (78.2) & $<0.05$ \\
\hline Bleeding between periods & $224(42.6)$ & $255(45.9)$ & 0.28 \\
\hline Frequent urination & $289(54.9)$ & $314(56.5)$ & 0.61 \\
\hline Discomfort during intercourse & $199(37.8)$ & $242(43.5)$ & 0.06 \\
\hline Pelvic pain requiring meds & $174(33.1)$ & $248(44.6)$ & $<0.05$ \\
\hline Pelvic pain not during periods & & & $<0.05$ \\
\hline Pelvic pain during periods & $48(9.1)$ & $86(15.5)$ & \\
\hline No pelvic pain & $357(67.9)$ & $311(55.9)$ & \\
\hline Current contraception & $390(74.1)$ & $383(68.8)$ & 0.05 \\
\hline $\begin{array}{l}\text { Previous medical condition (high blood pressure, } \\
\text { diabetes, asthma, thyroid problems, blood clots in legs) }\end{array}$ & $203(39.1)$ & $242(43.9)$ & 0.11 \\
\hline Marijuana & $51(9.8)$ & $42(7.7)$ & 0.23 \\
\hline Any prior UF treatment & $73(14.0)$ & $143(26.0)$ & $<0.05$ \\
\hline Regular, predictable menses & $368(71.0)$ & $381(69.7)$ & 0.62 \\
\hline Number of previous pregnancies & & & $<0.05$ \\
\hline 0 & $268(51.5)$ & $211(38.5)$ & \\
\hline 1 & $100(19.2)$ & $117(21.4)$ & \\
\hline 2 & $75(14.4)$ & $91(16.6)$ & \\
\hline 3 & $30(5.8)$ & $51(9.3)$ & \\
\hline 4 & $21(4.0)$ & $37(6.8)$ & \\
\hline 5 & $17(3.3)$ & $19(3.5)$ & \\
\hline 6 & $3(0.6)$ & $10(1.8)$ & \\
\hline$>6$ & $6(1.2)$ & $12(2.2)$ & \\
\hline Total UF volume $\left(\mathrm{cm}^{3}\right)$ & $158.6(24.9,418.2)$ & $152.1(39.2,368.4)$ & 0.96 \\
\hline Uterine volume $\left(\mathrm{cm}^{3}\right)$ & $305.6(137.0,654.6)$ & $462.2(225.0,885.5)$ & $<0.05$ \\
\hline Number of UFs measured & $2.0(1.0,3.0)$ & $2.0(1.0,4.0)$ & $<0.05$ \\
\hline Concern ${ }^{\mathrm{b}}$ & $45.8(20.0,76.6)$ & $35.0(15.0,65.0)$ & $<0.05$ \\
\hline Activities $^{\mathrm{b}}$ & $53.6(35.7,78.6)$ & $50.0(28.6,71.4)$ & $<0.05$ \\
\hline Energy/mood ${ }^{\mathrm{b}}$ & $50.0(32.1,75.0)$ & $46.4(25.0,67.9)$ & $<0.05$ \\
\hline Control $^{\mathrm{b}}$ & $50.0(30.0,70.0)$ & $50.0(25.0,70.0)$ & 0.58 \\
\hline Self-conscious ${ }^{\mathrm{b}}$ & $50.0(25.0,75.0)$ & $41.7(16.7,66.7)$ & $<0.05$ \\
\hline Sexual Function ${ }^{\mathrm{b}}$ & $50.0(25.0,75.0)$ & $50.0(25.0,87.5)$ & 0.37 \\
\hline Total-sum of 6 subscale scores above ${ }^{b}$ & $50.0(33.6,73.3)$ & $45.7(28.4,65.5)$ & $<0.05$ \\
\hline $\begin{array}{l}\text { Symptom severity (lower score indicates } \\
\text { more positive health status) }\end{array}$ & $50.0(34.4,67.2)$ & $53.1(34.4,75.0)$ & $<0.05$ \\
\hline Mobility & & & 0.20 \\
\hline I have no problems in walking about & $448(85.2)$ & 441 (79.9) & \\
\hline I have some problems in walking about & $47(8.9)$ & $63(11.4)$ & \\
\hline I have moderate problems in walking about & $23(4.4)$ & $32(5.8)$ & \\
\hline I have severe problems in walking about & $7(1.3)$ & $13(2.4)$ & \\
\hline I am confined to bed & $1(0.2)$ & $3(0.5)$ & \\
\hline Self-care & & & 0.32 \\
\hline I have no problems washing or dressing myself & $503(95.6)$ & $524(94.8)$ & \\
\hline I have slight problems washing or dressing myself & $17(3.2)$ & $18(3.3)$ & \\
\hline I have moderate problems washing or dressing myself & $6(1.1)$ & $7(1.3)$ & \\
\hline I have severe problems washing or dressing myself & $0(0.0)$ & $4(0.7)$ & \\
\hline I am unable to wash or dress myself & $0(0.0)$ & $0(0.0)$ & \\
\hline Usual activities & & & 0.19 \\
\hline I have no problems doing my usual activities & $348(66.3)$ & $382(69.2)$ & \\
\hline I have slight problems doing my usual activities & $103(19.6)$ & $94(17.0)$ & \\
\hline I have moderate problems doing my usual activities & $61(11.6)$ & $51(9.2)$ & \\
\hline I have severe problems doing my usual activities & $11(2.1)$ & $20(3.6)$ & \\
\hline I am unable to perform my usual activities & $2(0.4)$ & $5(0.9)$ & \\
\hline
\end{tabular}


TABle 4. (CONTINUED)

\begin{tabular}{lccc}
\hline Characteristic & White $(\mathrm{N}=526)$ & Black $(\mathrm{N}=557)$ & $\mathrm{p}^{\mathrm{a}}$ \\
\hline Pain/discomfort & & & $<0.05$ \\
I have no pain or discomfort & $124(23.6)$ & $150(27.1)$ & \\
I have slight pain or discomfort & $210(39.9)$ & $159(28.8)$ & \\
I have moderate pain or discomfort & $140(26.6)$ & $139(25.1)$ & \\
I have severe pain or discomfort & $41(7.8)$ & $70(12.7)$ & \\
I have extreme pain or discomfort & $11(2.1)$ & $35(6.3)$ & $<0.05$ \\
Anxiety/depression & $173(33.0)$ & $254(46.1)$ & \\
I am not anxious or depressed & $194(37.0)$ & $138(25.0)$ & \\
I am slightly anxious or depressed & $121(23.1)$ & $104(18.9)$ & \\
I am moderately anxious or depressed & $27(5.2)$ & $41(7.4)$ & \\
I am severely anxious or depressed & $9(1.7)$ & $14(2.5)$ & 0.65 \\
I am extremely anxious or depressed & $76.0(63.0,87.0)$ & $79.0(63.0,87.0)$ & \\
Visual Analogue Scale $(0-100)$ & & &
\end{tabular}

Data are median (interquartile range) or $n(\%)$.

${ }^{\mathrm{a}} p$-Values are based on Pearson chi-square tests for all categorical row variables. $p$-Values are based on chi-square rank based group means score statistics for all continuous/ordinal row variables. All tests treat the column variable as nominal.

${ }^{\mathrm{b}}$ Higher score indicates more positive health status.

health disparity for this disease. These evidence gaps make long-term follow-up of cohorts such as COMPARE-UF critical.

Evidence reports from the Agency for Healthcare Research and Quality have found almost no long-term $(>2$ years) evidence on comparative effectiveness of UF treatment options and have highlighted the massive knowledge gaps about UF treatments. ${ }^{26,27}$ The 2017 report included only 12 comparative studies of UF procedures, but 6 of them used hysterectomy as the reference group. ${ }^{28}$ Only three studies compared widely available uterine-sparing procedures with each other, and none included women in the United States, so results are not generalizable to our clinical population, where Black women incur the highest burden of UF. None of these studies had a follow-up time exceeding 2 years to assess clinical outcomes including treatment failure.

Nonetheless, most women who have either a hysterectomy or myomectomy to treat their UF will have improved short-term quality of life. In previous publications from the COMPARE-UF registry, ${ }^{7}$ we reported that at 6-12 weeks after treatment, although there were some differences in specific quality of life subscales, and some influence of procedure type and route of surgery (open vs. endoscopic), women who had surgical therapies had decreased symptoms and improved quality of life. ${ }^{9-11,15}$ More recently, we reported that overall, both women who had hysterectomy and those undergoing myomectomy had clinically meaningful ( $>10$ points) improvement in the UFS-QOL scores 1 year after the procedure. ${ }^{28}$

Stronger evidence is available for the long-term effectiveness of UAE. ${ }^{26,27}$ Based on seven randomized clinical trials studying nearly 8000 participants for up to 10 years following the procedure, there is strong quality of evidence that UAE results in decreased menstrual bleeding, volume reduction of UF, and moderate evidence of improved quality of life. ${ }^{26,27}$ Similar long-term studies for all uterine-sparing UF treatments and especially comparative effectiveness studies are critical to fully determine the effectiveness of all uterine-sparing procedures.

Limitations of this study include that COMPARE-UF was designed to assess the comparative effectiveness of the most common UF treatments rather than to understand the treatment decision-making process. We did not assess information on treatment preference, doctor counseling, or access. The data also focused on the procedure that was planned, which for only a small number of women changed during the actual procedure (Appendix Table A3). The participants are not representative of all women in the United States, as these participants had access to large health care institutions, many of which are considered centers of excellence for UF care, and COMPARE-UF had limited inclusion of women residing in rural locations. More than three-quarters of the women in these analyses also had private insurance. Therefore, by no means are we suggesting that the experience of the Black women and White women in this study are representative of the experiences of all Black women and White women; however, these data do describe the experience of some Black women and some White women and should serve as an impetus to improving our understanding of the interface between patients, clinical care, and health systems and providers. Strengths of the study include a large racially diverse study population from multiple clinical sites around the country, and the inclusion of a wide range of UF characteristics abstracted from imaging reports in medical record data.

Without prevention strategies, UF treatments will be needed on the present scale over the long term. Better understanding of the sources of racial differences in treatment choice, which do not appear to be owing to UF characteristics or other clinical characteristics, could both impact physician counseling of their patients as well as inform the development of future UF treatments. Given the extraordinarily high cumulative incidence of UF, these changes could lead to improved quality of life and health for most women in the United States. 
Table 5. Baseline Characteristics of Women With a Planned Uterine Artery Embolization

\begin{tabular}{|c|c|c|c|}
\hline Characteristic & White $(\mathrm{N}=57)$ & Black $(\mathrm{N}=196)$ & $\mathrm{p}^{\mathrm{a}}$ \\
\hline Age (years) & $47.0(42.0,49.0)$ & $45.0(41.0,47.0)$ & 0.05 \\
\hline Insurance & & & 0.48 \\
\hline Private & $45(80.4)$ & $165(84.2)$ & \\
\hline Active military & $0(0.0)$ & $3(1.5)$ & \\
\hline Other & $11(19.6)$ & $28(14.3)$ & \\
\hline BMI $\left(\mathrm{kg} / \mathrm{m}^{2}\right)$ & $27.3(24.3,29.9)$ & $31.3(25.9,36.9)$ & $<0.05$ \\
\hline Financial toxicity (lower score $=$ worse status) & $27.0(22.0,34.0)$ & $27.0(19.0,34.0)$ & 0.15 \\
\hline Duration of symptoms (years) & $3.0(1.0,7.0)$ & $6.0(2.0,13.0)$ & $<0.05$ \\
\hline Any bleeding symptoms & $48(84.2)$ & 179 (91.3) & 0.12 \\
\hline Menses $\geq 7$ days & $27(47.4)$ & $126(64.3)$ & $<0.05$ \\
\hline Heavy menses & $46(80.7)$ & $164(83.7)$ & 0.60 \\
\hline Bleeding between periods & $21(36.8)$ & $99(50.5)$ & 0.07 \\
\hline Frequent urination & $40(70.2)$ & 154 (78.6) & 0.19 \\
\hline Discomfort during intercourse & $20(35.1)$ & $87(44.4)$ & 0.21 \\
\hline Pelvic pain requiring meds & $25(43.9)$ & $99(50.5)$ & 0.38 \\
\hline Pelvic pain not during periods & & & 0.16 \\
\hline Pelvic pain during periods & $3(5.3)$ & $29(14.8)$ & \\
\hline No pelvic pain & $32(56.1)$ & $97(49.5)$ & \\
\hline Current contraception & $33(57.9)$ & $129(65.8)$ & 0.27 \\
\hline $\begin{array}{l}\text { Previous medical condition (high blood pressure, } \\
\text { diabetes, asthma, thyroid problems, blood clots in legs) }\end{array}$ & $26(45.6)$ & $118(61.1)$ & 0.04 \\
\hline Marijuana & $4(7.1)$ & $8(4.1)$ & 0.37 \\
\hline Any prior UF treatment & $8(14.0)$ & $38(19.7)$ & 0.33 \\
\hline Regular, predictable menses & $45(78.9)$ & $99(51.6)$ & $<0.05$ \\
\hline Number of previous pregnancies & & & 0.05 \\
\hline 0 & $19(33.3)$ & $38(19.7)$ & \\
\hline 1 & $6(10.5)$ & $31(16.1)$ & \\
\hline 2 & $14(24.6)$ & $40(20.7)$ & \\
\hline 3 & $12(21.1)$ & 34 (17.6) & \\
\hline 4 & $3(5.3)$ & $24(12.4)$ & \\
\hline 5 & $0(0.0)$ & $17(8.8)$ & \\
\hline 6 & $1(1.8)$ & $5(2.6)$ & \\
\hline$>6$ & $2(3.5)$ & $4(2.1)$ & \\
\hline Total UF volume $\left(\mathrm{cm}^{3}\right)$ & $131.6(69.4,346.7)$ & $133.8(45.0,307.3)$ & 0.30 \\
\hline Uterine volume $\left(\mathrm{cm}^{3}\right)$ & $507.2(320.1,915.6)$ & $542.9(311.5,867.8)$ & 0.91 \\
\hline Number of UFs measured & $3.0(1.0,4.0)$ & $2.0(1.0,4.0)$ & 0.90 \\
\hline Concern $^{\mathrm{b}}$ & $36.6(13.8,65.0)$ & $20.0(5.0,50.0)$ & $<0.05$ \\
\hline Activities $^{\mathrm{b}}$ & $46.4(28.6,75.0)$ & $39.3(17.9,57.1)$ & $<0.05$ \\
\hline Energy/mood ${ }^{\mathrm{b}}$ & $46.4(32.1,64.3)$ & $35.7(17.9,53.6)$ & $<0.05$ \\
\hline Control $^{b}$ & $45.0(30.0,65.0)$ & $40.0(20.0,65.0)$ & 0.57 \\
\hline Self-conscious ${ }^{\mathrm{b}}$ & $33.3(16.7,58.3)$ & $25.0(8.3,50.0)$ & 0.47 \\
\hline Sexual function ${ }^{b}$ & $50.0(25.0,75.0)$ & $37.5(12.5,75.0)$ & 0.11 \\
\hline Total (sum of 6 subscale scores above) ${ }^{\mathrm{b}}$ & $44.0(31.9,63.8)$ & $35.8(19.0,54.3)$ & $<0.05$ \\
\hline $\begin{array}{l}\text { Symptom severity (lower score indicates } \\
\text { more positive health status) }\end{array}$ & $62.5(46.9,71.9)$ & $68.8(50.0,81.3)$ & 0.06 \\
\hline Mobility & & & 0.33 \\
\hline I have no problems in walking about & $44(77.2)$ & $146(74.5)$ & \\
\hline I have some problems in walking about & $11(19.3)$ & $25(12.8)$ & \\
\hline I have moderate problems in walking about & $2(3.5)$ & $17(8.7)$ & \\
\hline I have severe problems in walking about & $0(0.0)$ & $6(3.1)$ & \\
\hline I am confined to bed & $0(0.0)$ & $2(1.0)$ & \\
\hline Self-care & & & 0.45 \\
\hline I have no problems washing or dressing myself & $52(91.2)$ & $184(93.9)$ & \\
\hline I have slight problems washing or dressing myself & $5(8.8)$ & $8(4.1)$ & \\
\hline I have moderate problems washing or dressing myself & $0(0.0)$ & $3(1.5)$ & \\
\hline I have severe problems washing or dressing myself & $0(0.0)$ & $1(0.5)$ & \\
\hline I am unable to wash or dress myself & $0(0.0)$ & $0(0.0)$ & \\
\hline Usual activities & & & 0.57 \\
\hline I have no problems doing my usual activities & $35(61.4)$ & $122(62.6)$ & \\
\hline I have slight problems doing my usual activities & $18(31.6)$ & $45(23.1)$ & \\
\hline I have moderate problems doing my usual activities & $3(5.3)$ & $21(10.8)$ & \\
\hline I have severe problems doing my usual activities & $1(1.8)$ & $5(2.6)$ & \\
\hline I am unable to perform my usual activities & $0(0.0)$ & $2(1.0)$ & \\
\hline
\end{tabular}


TABle 5. (CONTINUED)

\begin{tabular}{lccc}
\hline Characteristic & White $(\mathrm{N}=57)$ & Black $(\mathrm{N}=196)$ & $\mathrm{p}^{\mathrm{a}}$ \\
\hline Pain/discomfort & & & $<0.05$ \\
I have no pain or discomfort & $10(17.5)$ & $31(15.8)$ & \\
I have slight pain or discomfort & $26(45.6)$ & $58(29.6)$ & \\
I have moderate pain or discomfort & $20(35.1)$ & $54(27.6)$ & \\
I have severe pain or discomfort & $1(1.8)$ & $35(17.9)$ & \\
I have extreme pain or discomfort & $0(0.0)$ & $18(9.2)$ & \\
Anxiety/depression & $22(38.6)$ & $75(38.5)$ & \\
I am not anxious or depressed & $24(42.1)$ & $64(32.8)$ & \\
I am slightly anxious or depressed & $10(17.5)$ & $36(18.5)$ & \\
I am moderately anxious or depressed & $0(0.0)$ & $15(7.7)$ & \\
I am severely anxious or depressed & $1(1.8)$ & $5(2.6)$ & \\
I am extremely anxious or depressed & $79.0(67.5,87.0)$ & $76.0(59.0,88.0)$ & 0.86 \\
Visual Analogue Scale (0-100) & & &
\end{tabular}

Data are given as median (interquartile range) or $n(\%)$.

${ }^{a} p$-Values are based on Pearson chi-square tests for all categorical row variables. $p$-values are based on chi-square rank-based group means score statistics for all continuous/ordinal row variables. All tests treat the column variable as nominal.

${ }^{\mathrm{b}}$ Higher score indicates more positive health status.

Table 6. Odds of Black Women Versus White Women Having Uterine-Sparing TREATMENT Versus HySTERECTOMY

\begin{tabular}{|c|c|c|c|c|}
\hline & OR $(95 \% C I)$ & $\mathrm{p}$ & $\begin{array}{l}\text { Site-level variance } \\
\quad(95 \% \text { CI })\end{array}$ & $\mathrm{p}$ \\
\hline \multicolumn{5}{|l|}{ Uterine-sparing treatment is myomectomy or UAE } \\
\hline Race only & $1.62(1.36-1.94)$ & $<0.05$ & & \\
\hline $\begin{array}{l}\text { Race plus adjustment for site, demographics, } \\
\text { health, SES, }{ }^{a} \text { and symptoms }\end{array}$ & $2.72(1.94-3.81)$ & $<0.05$ & $2.54(0.99-15.32)$ & $<0.05$ \\
\hline \multicolumn{5}{|l|}{ Uterine-sparing treatment is myomectomy } \\
\hline Race only & $1.33(1.11-1.60)$ & $<0.05$ & & \\
\hline $\begin{array}{l}\text { Race plus adjustment for site, demographics, } \\
\text { health, SES, and symptoms }\end{array}$ & $2.41(1.63-3.56)$ & $<0.05$ & $0.82(0.36-3.33)$ & $<0.05$ \\
\hline \multicolumn{5}{|l|}{ Uterine-sparing treatment is $\mathrm{UAE}$} \\
\hline Race only & $4.32(3.12-5.99)$ & $<0.05$ & & \\
\hline $\begin{array}{l}\text { Race plus adjustment for site, demographics, } \\
\text { health, SES, and symptoms }\end{array}$ & $4.24(2.41-7.46)$ & $<0.05$ & $5.84(2.36-31.06)$ & $<0.05$ \\
\hline
\end{tabular}

Probability modeled is "event = uterine-sparing surgery"; reported ORs are for Black versus White.

${ }^{\text {a}}$ SES variables are insurance type and financial security.

$\mathrm{CI}$, confidence interval; ORs, odds ratios; SES, socioeconomic status.

\section{Data Sharing Agreement}

Data will be made publicly available by the DCRI in accordance with PCORI guidelines: https://www.pcori.org/about-us/ governance/policy-data-management-and-data-sharing.

\section{Disclaimer}

The content of this article is solely the responsibility of the authors, and readers should not interpret any statement in this product as an official position or the views of AHRQ, the U.S. Department of Health and Human Services, or PCORI.

\section{Acknowledgments}

The authors gratefully acknowledge the enrolling clinical centers and collaborators: Atlanta Fibroid Center of Atlanta
Interventional Institute: John C. Lipman, MD, Principal Investigator; Brigham and Women's and Affiliated Hospitals: R.M.A., Principal Investigator, Serene S. Srouji, MD, Antonio R. Gargiulo, MD (Massachusetts General Hospital), John C. Petrozza, MD (Beth Israel Deaconess Medical Center); University of California Fibroid Network: V.L.J., Principal Investigator (UC-San Francisco), Ram Parvataneni, MD, MPH (UC-Los Angeles), Erica Oberman, MD (UC-Los Angeles), Naghmeh Salamat Saberi, MD (UCIrvine), Shira Varon, MD (UC-San Diego), L. Elaine Waetjen, MD (UC-Davis); Henry Ford Health System: G.W., Principal Investigator; Inova Health: G.L.M., Principal Investigator, Abbas Shoberi, MD; Mayo Clinic: E.A.S., Principal Investigator, S.L.-T., Bijan Borah, PhD, Joyce Balls-Berry, PhD; Satellite Site Investigators: Jennifer Bantz, MD, Paul Matigbay, MD, Gokhan Anil, MD, Jason Dewitt, MD; Michigan Medicine (University of 
Michigan): E.E.M., Principal Investigator; University of Mississippi Medical Center: K.W., Principal Investigator, J. Preston Parry, MD; UNC Health Care (The University of North Carolina at Chapel Hill): W.K.N., Principal Investigator, Andrea Knittel, MD, A.I.V., Lauren Schiff, MD, Stephen Loehr, MD.

\section{Author Disclosure Statement}

G.W., W.K.N., S.Z., F.L., L.T., J.B.S., S.V., R.M.A., G.L.M., A.I.V., L.A.W. and K.W. have nothing to disclose. E.A.S. reports personal fees from Bayer, AbbVie, Myovant, ObsEva, UpToDate, Med Learning Group, PeerView, PER and Massachusetts Medical Society outside the submitted work and an issued patent "Methods and Compounds for Treatment of Abnormal Uterine Bleeding" 6440445. S.L.-T. reports grants from Bayer and personal fees from Allergan Pharma and UpToDate outside the submitted work. M.P.D. reports grants from AbbVie, Bayer, and ObsEva outside the submitted work. E.E.M. reports consultant work for Allergan and Myovant Sciences outside the submitted work. E.R.M. reports personal fees from AbbVie, Merck, Allergan, and Bayer outside the submitted work. V.L.J. reports grants from Acessa Health outside the submitted work.

\section{Funding Information}

This study was supported by Grant No. P50HS023418 from the Agency for Healthcare Research and Quality (AHRQ) with funding provided by the Patient-Centered Outcomes Research Institute (PCORI) under Memorandum of Understanding No. 2013-001.

\section{References}

1. Baird DD, Dunson DB, Hill MC, Cousins D, Schectman JM. High cumulative incidence of uterine leiomyoma in black and white women: Ultrasound evidence. Am J Obstet Gynecol 2003;188:100-107.

2. Al-Hendy A, Myers ER, Stewart E. Uterine fibroids: Burden and unmet medical need. Semin Reprod Med 2017;35: 473-480.

3. Metwally M, Raybould G, Cheong YC, Horne AW. Surgical treatment of fibroids for subfertility. Cochrane Database Syst Rev 2020;1:CD003857.

4. Stewart EA, Laughlin-Tommaso SK, Catherino WH, Lalitkumar S, Gupta D, Vollenhoven B. Uterine fibroids. Nat Rev Dis Primers 2016;2:16043.

5. Kjerulff KH, Langenberg P, Seidman JD, Stolley PD, Guzinski GM. Uterine leiomyomas. Racial differences in severity, symptoms and age at diagnosis. J Reprod Med 1996; 41:483-490.

6. Stewart EA, Nicholson WK, Bradley L, Borah BJ. The burden of uterine fibroids for African-American women: Results of a national survey. J Womens Health (Larchmt) 2013;22:807-816.

7. Stewart EA, Lytle BL, Thomas L, et al. The comparing options for management: Patient-centered results for uterine fibroids (COMPARE-UF) registry: Rationale and design. Am J Obstet Gynecol 2018;219:95.e1e95.e10.
8. Laughlin-Tommaso SK, Jacoby VL, Myers ER. Disparities in fibroid incidence, prognosis, and management. Obstet Gynecol Clin North Am 2017;44:81-94.

9. Coyne KS, Margolis MK, Bradley LD, Guido R, Maxwell GL, Spies JB. Further validation of the uterine fibroid symptom and quality-of-life questionnaire. Value Health 2012;15:135-142.

10. Harding G, Coyne KS, Thompson CL, Spies JB. The responsiveness of the uterine fibroid symptom and healthrelated quality of life questionnaire (UFS-QOL). Health Qual Life Outcomes 2008;6:99.

11. Spies JB, Coyne K, Guaou Guaou N, Boyle D, SkyrnarzMurphy K, Gonzalves SM. The UFS-QOL, a new disease-specific symptom and health-related quality of life questionnaire for leiomyomata. Obstet Gynecol 2002;99:290-300.

12. Herdman M, Gudex C, Lloyd A, et al. Development and preliminary testing of the new five-level version of EQ-5D (EQ-5D-5L). Qual Life Res 2011;20:1727-1736.

13. de Souza JA, Yap BJ, Wroblewski K, et al. Measuring financial toxicity as a clinically relevant patient-reported outcome: The validation of the COmprehensive Score for financial Toxicity (COST). Cancer 2017;123:476-484.

14. Kroenke K, Spitzer RL, Williams JB. The Patient Health Questionnaire-2: Validity of a two-item depression screener. Med Care 2003;41:1284-1292.

15. Nicholson WK, Wegienka G, Zhang S, et al. Short-term health-related quality of life after hysterectomy compared with myomectomy for symptomatic leiomyomas. Obstet Gynecol 2019;134:261-269.

16. Laughlin-Tommaso SK, Lu D, Thomas L, et al. Short-term quality of life after myomectomy for uterine fibroids from the COMPARE-UF Fibroid Registry. Am J Obstet Gynecol 2020;222:345.e1-345.e22.

17. Garver KL, Garver B. Eugenics: Past, present, and the future. Am J Hum Genet 1991;49:1109-1118.

18. Roberts DE. Killing the black body: Race, reproduction, and the meaning of liberty. New York: Vintage Books, 1997.

19. Farquhar CM, Harvey SA, Yu Y, Sadler L, Stewart AW. A prospective study of 3 years of outcomes after hysterectomy with and without oophorectomy. Am J Obstet Gynecol 2006;194:711-717.

20. Leppert PC, Legro RS, Kjerulff KH. Hysterectomy and loss of fertility: Implications for women's mental health. J Psychosom Res 2007;63:269-274.

21. Augustus CE. Beliefs and perceptions of African American women who have had hysterectomy. J Transcult Nurs 2002; 13:296-302.

22. Bossick AS, Sangha R, Olden H, Alexander GL, Wegienka G. Identifying what matters to hysterectomy patients: Postsurgery perceptions, beliefs, and experiences. J Patient Cent Res Rev 2018;5:167-175.

23. Williams RD, Clark AJ. A qualitative study of women's hysterectomy experience. J Womens Health Gend Based Med 2000;9(Suppl 2):S15-S25.

24. Bulun SE. Uterine fibroids. N Engl J Med 2013;369:13441355.

25. Peddada SD, Laughlin SK, Miner K, et al. Growth of uterine leiomyomata among premenopausal black and white women. Proc Natl Acad Sci U S A 2008;105:1988719892.

26. Myers ER, Barber MD, Gustilo-Ashby T, Couchman G, Matchar DB, McCrory DC. Management of uterine leio- 
myomata: What do we really know? Obstet Gynecol 2002; 100:8-17.

27. Hartmann KE, Fonnesbeck C, Surawicz T, et al. Management of uterine fibroids. Comparative Effectiveness Review No. 195. Rockville, MD: Agency for Healthcare Research and Quality, 2017.

28. Wallace K, Zhang S, Thomas L, et al. Comparative effectiveness of hysterectomy versus myomectomy on one-year health-related quality of life in women with uterine fibroids. Fertil Steril 2020;113:618-626.
Address correspondence to:

Ganesa Wegienka, PhD

Department of Public Health Sciences

Henry Ford Health System

1 Ford Place, $3 E$

Detroit, MI 48202

USA

E-mail: gwegien1@hfhs.org

\section{Appendix}

Appendix Table A1. Odds of Black Women Versus White Women

Having Uterine-Sparing Treatment Versus Hysterectomy

\begin{tabular}{|c|c|c|c|c|}
\hline & OR $(95 \% C I)$ & $\mathrm{p}$ & $\begin{array}{c}\text { Site-level } \\
\text { variance }(95 \% \text { CI })\end{array}$ & $\mathrm{p}$ \\
\hline \multicolumn{5}{|c|}{ Uterine-sparing treatment is myomectomy or UAE or EA or MRgFUS } \\
\hline Race only & $1.50(1.26-1.78)$ & $<0.05$ & & \\
\hline $\begin{array}{l}\text { Race plus adjustment for site, demographics, health, } \\
\text { SES, }{ }^{\text {a }} \text { and symptoms }\end{array}$ & $2.26(1.65-3.09)$ & $<0.05$ & $2.43(0.95-14.51)$ & $<0.05$ \\
\hline
\end{tabular}

Probability modeled is "event=uterine-sparing surgery"; reported ORs are for Black versus White.

${ }^{a}$ SES variables are insurance type and financial toxicity.

CI, confidence interval; EA, endometrial ablation; MRgFUS, MRI-guided focused ultrasound; ORs, odds ratios; SES, socioeconomic status; UAE, uterine artery embolization.

Appendix Table A2. Baseline Patient Characteristics by Race

\begin{tabular}{|c|c|c|c|}
\hline Characteristic & White $(\mathrm{N}=1033)$ & Black $(\mathrm{N}=1111)$ & $\mathrm{p}^{\mathrm{a}}$ \\
\hline Age (years) & $42.0(36.0,47.0)^{\mathrm{a}}$ & $41.0(36.0,46.0)^{\mathrm{a}}$ & $<0.05$ \\
\hline Insurance & & & $<0.05$ \\
\hline Private & $913(88.7)$ & $858(77.4)$ & \\
\hline Active military & $4(0.4)$ & $15(1.4)$ & \\
\hline Other & $112(10.9)$ & $235(21.2)$ & \\
\hline BMI $\left(\mathrm{kg} / \mathrm{m}^{2}\right)$ & $26.6(23.0,31.8)$ & $31.4(26.7,37.2)$ & $<0.05$ \\
\hline Financial toxicity (lower score $=$ worse status) & $29.0(22.0,36.0)$ & $26.0(17.0,32.0)$ & $<0.05$ \\
\hline Duration of symptoms (years) & $3.0(1.0,6.0)$ & $5.0(2.0,11.0)$ & $<0.05$ \\
\hline Any bleeding symptoms & $832(80.8)$ & $947(85.4)$ & $<0.05$ \\
\hline Menses $\geq 7$ days & $548(53.2)$ & $672(60.6)$ & $<0.05$ \\
\hline Heavy menses & $768(74.6)$ & $883(79.6)$ & $<0.05$ \\
\hline Bleeding between periods & $477(46.3)$ & $545(49.1)$ & 0.19 \\
\hline Frequent urination & $592(57.5)$ & $686(61.9)$ & $<0.05$ \\
\hline Discomfort during intercourse & $412(40.0)$ & $503(45.4)$ & $<0.05$ \\
\hline Pelvic pain requiring meds & $408(39.6)$ & $548(49.4)$ & $<0.05$ \\
\hline Pelvic pain not during periods & & & $<0.05$ \\
\hline Pelvic pain during periods & $117(11.4)$ & $163(14.7)$ & \\
\hline No pelvic pain & $630(61.2)$ & $568(51.2)$ & \\
\hline Current contraception & $736(71.2)$ & $736(66.2)$ & $<0.05$ \\
\hline $\begin{array}{l}\text { Previous medical condition (high blood pressure, } \\
\text { diabetes, asthma, thyroid problems, blood clots in legs) }\end{array}$ & $453(44.5)$ & $577(52.6)$ & $<0.05$ \\
\hline Marijuana & $82(8.1)$ & $77(7.0)$ & $<0.05$ \\
\hline Any prior treatment & $163(15.9)$ & $264(24.1)$ & $<0.05$ \\
\hline Regular, predictable menses & $676(66.3)$ & $648(59.6)$ & $<0.05$ \\
\hline Number of pregnancies & & & $<0.05$ \\
\hline 0 & $407(39.8)$ & $302(27.6)$ & \\
\hline 1 & $168(16.4)$ & $192(17.6)$ & \\
\hline 2 & $187(18.3)$ & $191(17.5)$ & \\
\hline 3 & $123(12.0)$ & $148(13.5)$ & \\
\hline 4 & $62(6.1)$ & $124(11.3)$ & \\
\hline
\end{tabular}


Appendix Table A2. (Continued)

\begin{tabular}{|c|c|c|c|}
\hline Characteristic & White $(\mathrm{N}=1033)$ & Black $(\mathrm{N}=1111)$ & $\mathrm{p}^{\mathrm{a}}$ \\
\hline 5 & $40(3.9)$ & $66(6.0)$ & \\
\hline 6 & $15(1.5)$ & $31(2.8)$ & \\
\hline$>6$ & $20(2.0)$ & 39 (3.6) & \\
\hline Total UF volume $\left(\mathrm{cm}^{3}\right)$ & $140.5(28.6,364.8)$ & $147.3(40.6,336.7)$ & 0.27 \\
\hline Uterine volume $\left(\mathrm{cm}^{3}\right)$ & $324.2(159.1,640.0)$ & $492.5(255.8,920.7)$ & $<0.05$ \\
\hline Number of UFs measured & $2.0(1.0,3.0)$ & $2.0(1.0,4.0)$ & $<0.05$ \\
\hline Concern ${ }^{\mathrm{b}}$ & $40.0(20.0,71.6)$ & $30.0(10.0,55.0)$ & $<0.05$ \\
\hline Activities ${ }^{b}$ & $50.0(28.6,75.0)$ & $42.9(21.4,67.9)$ & $<0.05$ \\
\hline Energy $/ \mathrm{mood}^{\mathrm{b}}$ & $50.0(28.9,71.4)$ & $42.9(21.4,60.7)$ & $<0.05$ \\
\hline Control $^{\mathrm{b}}$ & $50.0(30.0,70.0)$ & $45.0(25.0,70.0)$ & $<0.05$ \\
\hline Self-conscious ${ }^{\mathrm{b}}$ & $41.7(16.7,66.7)$ & $33.3(8.3,66.7)$ & $<0.05$ \\
\hline Sexual function ${ }^{b}$ & $50.0(25.0,75.0)$ & $50.0(12.5,75.0)$ & 0.27 \\
\hline Total (sum of six subscale scores above) ${ }^{\mathrm{b}}$ & $47.4(31.0,68.1)$ & $40.5(22.4,60.3)$ & $<0.05$ \\
\hline $\begin{array}{l}\text { Symptom severity (lower score indicates } \\
\text { more positive health status) }\end{array}$ & $56.3(37.5,71.9)$ & $62.5(40.6,78.1)$ & $<0.05$ \\
\hline Mobility & & & $<0.05$ \\
\hline I have no problems in walking about & $852(82.6)$ & $819(74.4)$ & \\
\hline I have some problems in walking about & $114(11.1)$ & $152(13.8)$ & \\
\hline I have moderate problems in walking about & $51(4.9)$ & $91(8.3)$ & \\
\hline I have severe problems in walking about & $13(1.3)$ & $33(3.0)$ & \\
\hline I am confined to bed & $1(0.1)$ & $6(0.5)$ & \\
\hline Self-care & & & 0.02 \\
\hline I have no problems washing or dressing myself & $978(95.0)$ & $1029(93.2)$ & \\
\hline I have slight problems washing or dressing myself & $39(3.8)$ & $45(4.1)$ & \\
\hline I have moderate problems washing or dressing myself & $12(1.2)$ & $21(1.9)$ & \\
\hline I have severe problems washing or dressing myself & $0(0.0)$ & $8(0.7)$ & \\
\hline I am unable to wash or dress myself & $1(0.1)$ & $1(0.1)$ & \\
\hline Usual activities & & & 0.08 \\
\hline I have no problems doing my usual activities & $665(64.5)$ & $708(64.3)$ & \\
\hline I have slight problems doing my usual activities & $215(20.9)$ & $215(19.5)$ & \\
\hline I have moderate problems doing my usual activities & 120 (11.6) & 119 (10.8) & \\
\hline I have severe problems doing my usual activities & $26(2.5)$ & $45(4.1)$ & \\
\hline I am unable to perform my usual activities & $5(0.5)$ & $14(1.3)$ & \\
\hline Pain/discomfort & & & $<0.05$ \\
\hline I have no pain or discomfort & $213(20.7)$ & $229(20.7)$ & \\
\hline I have slight pain or discomfort & $387(37.5)$ & $288(26.1)$ & \\
\hline I have moderate pain or discomfort & 315 (30.6) & $324(29.3)$ & \\
\hline I have severe pain or discomfort & $93(9.0)$ & $179(16.2)$ & \\
\hline I have extreme pain or discomfort & $23(2.2)$ & $84(7.6)$ & \\
\hline Anxiety/depression & & & $<0.05$ \\
\hline I am not anxious or depressed & $353(34.4)$ & $475(43.1)$ & \\
\hline I am slightly anxious or depressed & $388(37.8)$ & $300(27.2)$ & \\
\hline I am moderately anxious or depressed & $222(21.6)$ & 205 (18.6) & \\
\hline I am severely anxious or depressed & $50(4.9)$ & $85(7.7)$ & \\
\hline I am extremely anxious or depressed & $14(1.4)$ & $37(3.4)$ & \\
\hline Visual analog scale $(0-100)$ & $75.0(60.0,85.0)$ & $75.0(60.0,86.0)$ & 0.17 \\
\hline
\end{tabular}

Data are given s median (interquartile range) or $n(\%)$.

${ }^{a} p$-Values are based on Pearson chi-square tests for all categorical row variables. $p$-Values are based on chi-square rank-based group means score statistics for all continuous/ordinal row variables. This is equivalent to Wilcoxon tests. All tests treat the column variable as nominal.

${ }^{\mathrm{b}}$ Higher score indicates more positive health status.

BMI, body mass index; UF, uterine fibroid.

Appendix Table A3. Planned Treatment Versus Completed Treatment

\begin{tabular}{lrrrrrrr}
\hline & \multicolumn{7}{c}{ Actual treatment performed } \\
\cline { 2 - 8 } & Unknown & Myo & Hyst & UAE & EA & RFA & IUD \\
\hline Planned treatment & & & & & & & \\
Myo & 19 & $\mathbf{1 0 4 2}$ & 18 & 0 & 4 & 0 & 0 \\
Hyst & 8 & 5 & $\mathbf{7 9 5}$ & 0 & 0 & 0 & 0 \\
UAE & 34 & 0 & 0 & $\mathbf{2 1 9}$ & 0 & 0 & 0 \\
\hline
\end{tabular}

Values in bold are concordant planned and completed treatments.

Planned treatment is based on participant report, and completed treatment is from medical chart review.

Hyst, hysterectomy; IUD, intrauterine device; Myo, myomectomy; RFA, radiofrequency ablation. 